\title{
Thyroid-Stimulating Hormone-Secreting Pituitary Adeno- mas : Single Institutional Experience of 14 Consecutive Cases
}

\author{
Joonho Byun, Jeong Hoon Kim, Young-Hoon Kim, Young Hyun Cho, Seok Ho Hong, Chang Jin Kim \\ Department of Neurological Surgery, Asan Medical Center, University of Ulsan College of Medicine, Seoul, Korea
}

Objective : Thyroid-stimulating hormone (TSH)-secreting pituitary adenoma (PA) is an extremely rare functioning form of PA that accounts for $0.7-2 \%$ of all such cases. The previously reported outcomes of the surgical removal of TSH-PA are poor. Owing to its extremely low incidence, most available reports on TSH-PA are case reports or small case series. Thus, we investigated the clinical and endocrinological outcomes of surgically treated TSH-PA through our institutional series.

Methods : We retrospectively reviewed 14 consecutive cases of surgically treated TSH-PA, focusing on the clinical, radiological, surgical, and endocrinological data.

Results : There were seven male (50\%) and seven female (50\%) patients. The mean age was 42.5 years (range, 19-63). The mean tumor size was $16.6 \mathrm{~mm}$ (range, 4-30). Optic chiasm compression was noted in six patients (42.9\%), and no patient showed cavernous sinus invasion. Thirteen of 14 patients (92.8\%) underwent transnasal transsphenoidal approach (TSA), and one patient underwent TSA followed by transcranial approach for residual tumor removal. Thirteen of 14 patients (92.8\%) showed endocrinological remission; all patients who experienced remission showed subnormal levels of TSH $(<0.4 \mu \mathrm{U} / \mathrm{mL})$ on postoperative day 2. Recurrence occurred in two patients (14.2\%). One patient underwent subsequent revision transnasal TSA for recurrent tumor removal, and the other patient underwent gamma knife radiosurgery for recurrence.

Conclusion : Surgical treatment showed excellent surgical outcomes. The TSH level in the immediate postoperative period may be a predictor for endocrinological remission.

Key Words : Thyroid-stimulating hormone · Pituitary adenoma · Surgery.

\section{INTRODUCTION}

Pituitary adenoma (PA) is the third most common primary brain tumor ${ }^{14)}$. The incidence of functioning PAs is approximately $46 \%$, and the most common functioning PA is prolac- tinoma $^{18)}$. Thyroid-stimulating hormone (TSH)-secreting PA is an extremely rare form of functioning PA that accounts for $0.7-2 \%$ of all cases ${ }^{13,18)}$. Recently, the widespread use of brain magnetic resonance imaging (MRI) has made the early diagnosis of TSH-PA easier and thus has increased its reported in-

\footnotetext{
- Received : August 5, $2019 \bullet$ Revised : September 11, 2019 •Accepted : October 6, 2019

- Address for reprints : Jeong Hoon Kim

Department of Neurological Surgery, Asan Medical Center, University of Ulsan College of Medicine, 88 Olympic-ro 43-gil, Songpa-gu, Seoul 05505, Korea Tel : +82-2-3010-3559, Fax : +82-2-476-6738, E-mail : jhkim1@amc.seoul.kr, ORCID : https://orcid.org/0000-0001-7385-2368
} 
cidence $^{13,20)}$. Surgical removal of the pituitary tumor is considered to be the initial treatment of choice for functioning PA excluding prolactinoma. However, the previously reported outcomes of the surgical removal of TSH-PA are poor, and the rate of endocrinological remission of TSH-PA was reported to be $35-84 \%^{3,7,17,20,21)}$. Currently, surgical treatment alone for TSH-PA has not been recognized as a single effective treatment. In addition, owing to the extreme rarity of TSH-PA, most available reports on the radiological and endocrinological outcomes of TSH-PA after surgery are case reports or small case series. Thus, we here investigated the clinical and endocrinological outcomes of surgically treated TSH-PA through our institutional series. We believe that this study makes a valuable addition to the clinical results on TSH-PA.

\section{MATERIALS AND METHODS}

\section{Patients}

This study was approved by the Institutional Review Board of Asan Medical Center (No. 2018-1505). Our institutional database was searched for patients who underwent surgery and were diagnosed with TSH-PA between January 1996 and April 2018. Fourteen patients treated consecutively were analyzed for their clinical outcomes including long-term postoperative endocrinological remission and recurrence. All patients were referred to us with the proven diagnosis of TSH-PA for surgical treatment. Seven of the 14 patients (50\%) had received medical therapy to control the hyperthyroidism symptoms or to minimize perioperative cardiovascular complications.

\section{Preoperative and postoperative laboratory tests}

In accordance with the consensus of the European Thyroid
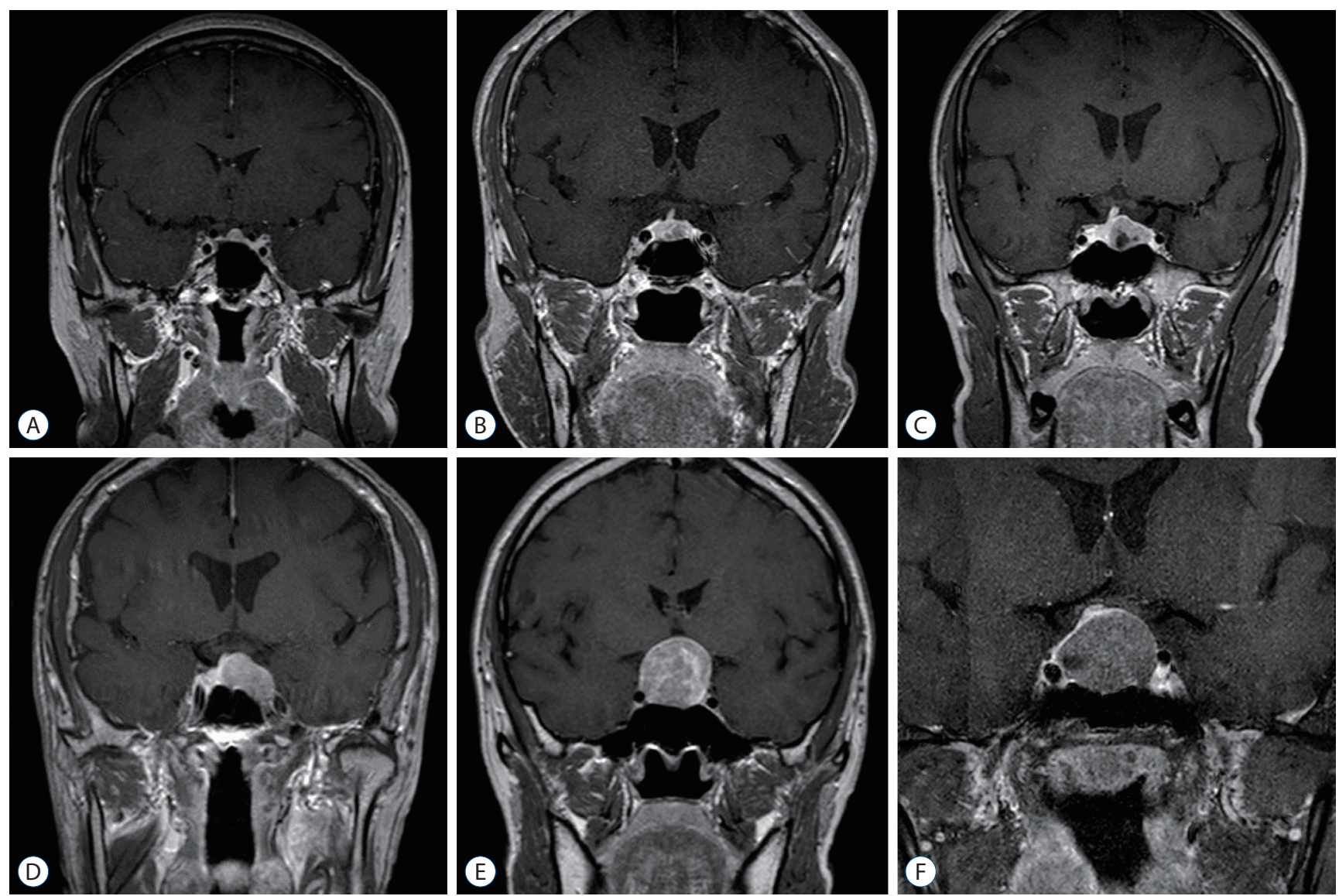

Fig. 1. Various radiological findings of TSH-secreting pituitary adenomas. A-F : Various shapes and sizes of TSH-PA tumors. All of these patients underwent transnasal transsphenoidal surgery and experienced endocrinological remission. TSH : thyroid-stimulating hormone, PA : pituitary adenoma. 
Association, TSH-PA was diagnosed if high concentrations of T3 and T4 were present with non-suppressed levels of TSH and a pituitary lesion identified on brain $\mathrm{MRI}^{1)}$. The levels of TSH, T3, free T4 (fT4), adrenocorticotropic hormone, growth hormone, follicle-stimulating hormone/luteinizing hormone, prolactin, and cortisol were measured. All blood samples were obtained in the preoperative period to establish the endocrinological diagnosis. The levels of TSH, T3, and fT4 were measured on 2 days after surgery (POD2) and every month after that. If patients showed other hormonal deficiency symptoms, an additional hormonal test was performed. After establishing endocrinological remission, TSH, T3, and fT4 levels were checked annually. Endocrinological remission was confirmed when TSH levels decreased to the subnormal range in the immediate postoperative period with normalization of T3 and fT4 levels. Thyroid hormones including TSH, fT4, and T3 were measured using Microlab AT+2 (Automatic pipetting system; Hamilton Inc., Reno, NV, USA) and RIA-MAT 280 (Full-automatic RIA system; Stratec Inc., Birkenfeld, Germany). The ranges of TSH of 0.4 to $5.0 \mu \mathrm{U} / \mathrm{mL}$, fT4 of $0.8-1.9 \mathrm{ng} / \mathrm{dL}$, and T3 of 81-197 ng/ $\mathrm{dL}$ were considered to be normal.

\section{Surgery and follow-up}

All surgical procedures including the approaches followed, extent of resection, and complications were reviewed using electronic medical records. Cavernous sinus invasion was evaluated using the revised Knosp classification system ${ }^{6,8)}$. Procedures were performed by specialized pituitary neurosurgeons (CJK and JHK) via a microscopic transnasal transsphenoidal approach (TSA). One patient underwent an additional transcranial approach (TCA) via a left pterional craniotomy. Postoperative brain MRI was performed 3 months after surgery and the images were checked to determine whether endocrinological recurrence had occurred.

Recurrence-free survival of our patients was analyzed using Kaplan-Meier survival analysis. All statistical analyses were conducted using PASW Statistics for Windows, version 18.0 (SPSS Inc., Chicago, IL, USA).

\section{RESULTS}

There were seven male (50\%) and seven female (50\%) patients. The mean age was 42.5 years (range, 19-63). The most common presenting symptoms were fatigue $(4,28.6 \%)$ and palpitation (4, 28.6\%). The mean tumor size was $16.6 \mathrm{~mm}$ (range, 4-30). Optic chiasm compression was noted in six patients (42.9\%). There were three patients (21.4\%) with microadenoma and $11(78.6 \%)$ with macroadenoma. Three patients (21.4\%) showed revised Knosp classification grade 0, three patients (21.4\%) showed grade I, seven patients (50\%) showed grade II, one patient (7.1\%) showed grade IIIA; there was no patient showing grade IV. There was no feature indicative of a differential diagnosis of TSH-PA from non-functioning and functioning PA on brain MRI. Various MRI findings are presented in Fig. 1. Representative case's MRI findings are presented in Fig. 2 and histopathologic findings are presented in Fig. 3.

Thirteen of the 14 patients (92.8\%) underwent transnasal TSA, and one patient underwent subtotal removal through transnasal TSA and subsequent TCA for residual tumor removal (Fig. 2). Seven of the 14 patients (50\%) received preoperative medical treatment to control hyperthyroidism symptoms;
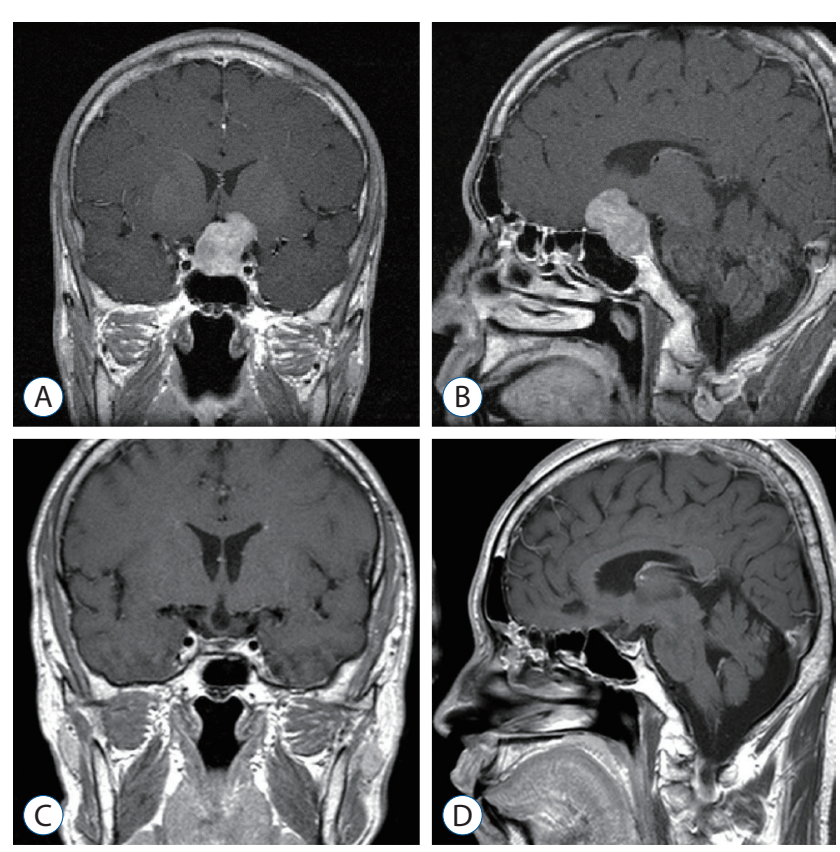

Fig. 2. Representative case of TSH-secreting pituitary adenomas. In a 33-year-old male patient, the tumor grew in the suprasellar area and extended to the anterior cranial fossa. The patient underwent combined transnasal transsphenoidal surgery followed by transcranial surgery via left pterional craniotomy. Postoperative endocrinological remission was achieved (A and B : preoperative sagittal and coronal T1-weighted enhanced MRI image, $C$ and D : postoperative coronal and sagittal T1weighted enhanced MRI image). TSH : thyroid-stimulating hormone, MRI : magnetic resonance imaging. 
among these patients, six were treated with methimazole and one was treated with propylthiouracil. The median follow-up period was 94.2 months. Detailed characteristics of the enrolled patients are described in Table 1.

Thirteen of the 14 patients $(92.8 \%)$ showed endocrinological remission. All patients who experienced remission showed subnormal levels of TSH on postoperative day 2 (less than 0.4 $\mu \mathrm{U} / \mathrm{mL})$. There was no patient with postoperative permanent diabetes insipidus (DI); however, five of 14 patients (35.7) needed short-term replacement of vasopressin for temporary DI. No patient needed postoperative hormonal replacement, and there was no postoperative complication.

Recurrence occurred in two patients (14.2\%). One patient (case \#5 in Table 2) showed radiological recurrence on brain MRI and underwent subsequent revision transnasal TSA for recurrent tumor removal. This patient experienced hormonal remission after revision surgery. The other patient (case \#6 in Table 2) underwent gamma knife radiosurgery (GKRS) (dose, $30 \mathrm{~Gy} / 50 \%$ isodose line) for recurrence. After hormonal recurrence, thyroid hormone level measurements with alpha subunit laboratory test as well as thyroid ultrasonography and brain MRI were performed. There were increased levels of
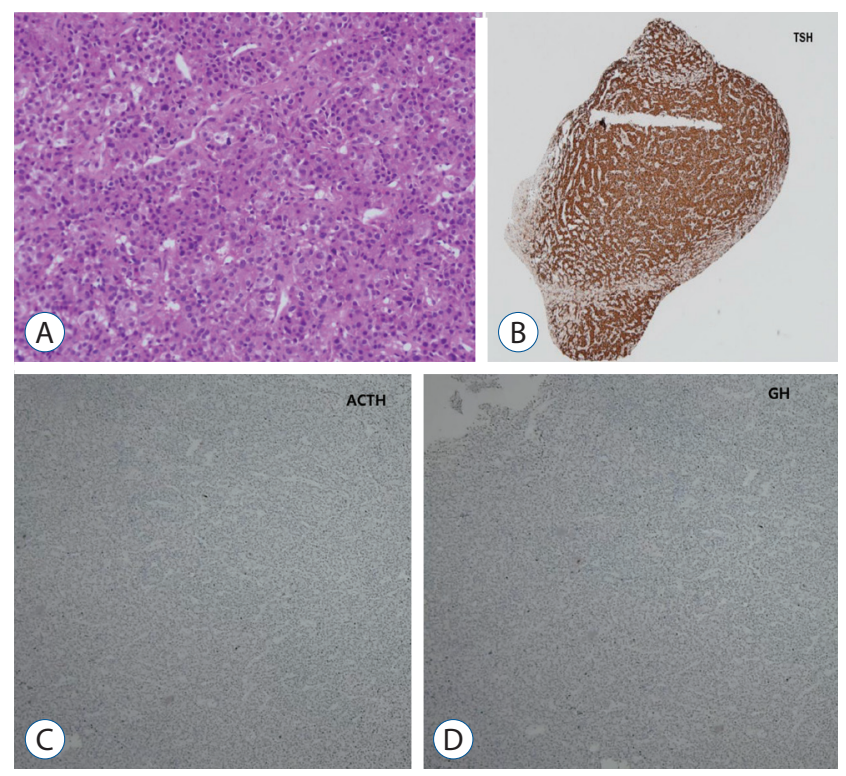

Fig. 3. A : Monomorphic cell with granular cytoplasm, round nuclei, and dispersed chromatin are present in the adenoma (Hematoxylin-Eosin staining, $\times 100$ ). B-D : Immunostaining for TSH, ACTH, GH, and tumor showed positivity for TSH ( $\mathrm{B}$ : TSH immuostaining, $\times 4$; $\mathrm{C}: \mathrm{ACTH}$ immunostaining, $\times 40$; D : GH immunostaining, $\times 40$ ). TSH : thyroidstimulating hormone, $\mathrm{ACTH}$ : adrenocorticotropic hormone, $\mathrm{GH}$ : growth hormone.
Table 1. Patient demographics of TSH-secreting pituitary adenoma

\begin{tabular}{|c|c|}
\hline Variable & Value $(n=14)$ \\
\hline Age (years) & $42.5(19-63)$ \\
\hline \multicolumn{2}{|l|}{ Sex } \\
\hline Male & $7(50)$ \\
\hline Female & $7(50)$ \\
\hline \multicolumn{2}{|l|}{ Presenting symptoms } \\
\hline Fatigue & $4(28.6)$ \\
\hline Palpitation & $4(28.6)$ \\
\hline Heat intolerance & $1(7.1)$ \\
\hline Headache & $1(7.1)$ \\
\hline Visual disturbance & $1(7.1)$ \\
\hline Thyrotoxic periodic paralysis & $1(7.1)$ \\
\hline Incidental finding (medical check-up) & $2(14.3)$ \\
\hline Tumor size $(\mathrm{mm})$ & $16.6(4-30)$ \\
\hline Microadenoma & $3(21.4)$ \\
\hline Macroadenoma & $11(78.6)$ \\
\hline Optic chiasm compression & $6(42.9)$ \\
\hline \multicolumn{2}{|l|}{ Cavernous sinus involvement } \\
\hline \multicolumn{2}{|l|}{ Knosp classification system } \\
\hline Grade 0 & $3(21.4)$ \\
\hline Grade I & $3(21.4)$ \\
\hline Grade II & $7(50.0)$ \\
\hline Grade III & $1(7.1)$ \\
\hline Grade IIIA & 1 \\
\hline Grade IV & 0 \\
\hline \multicolumn{2}{|l|}{ Combined disease } \\
\hline Hypertension & $3(21.4)$ \\
\hline Atrial fibrillation & $1(7.1)$ \\
\hline \multicolumn{2}{|l|}{ Surgery } \\
\hline Transsphenoidal surgery alone & $12(92.3)$ \\
\hline Transcranial surgery alone & 0 \\
\hline Transsphenoidal+transcranial surgery & $1(7.7)$ \\
\hline Preoperative medical therapy & $7(50.0)$ \\
\hline \multicolumn{2}{|l|}{ Regimen } \\
\hline Methimazole with/without Lugol's solution & $6(42.9)$ \\
\hline Propylthiouracil & $1(7.1)$ \\
\hline Duration of free T4 normalization (days) & $67.5(2-150)$ \\
\hline Endocrinological remission & 13/14 (92.8) \\
\hline Symptomatic resolution & $8 / 10(80)$ \\
\hline \multicolumn{2}{|l|}{ Postoperative DI } \\
\hline Temporary DI & $5(35.7)$ \\
\hline Permanent DI & 0 \\
\hline Postoperative hormonal replacement & 0 \\
\hline Postoperative complications & 0 \\
\hline Recurrence & $2(14.2)$ \\
\hline \multicolumn{2}{|l|}{ Treatment } \\
\hline \multicolumn{2}{|l|}{ Redo-surgery } \\
\hline Transsphenoidal surgery & $1(7.1)$ \\
\hline \multicolumn{2}{|l|}{ Radiosurgery } \\
\hline Gamma-knife radiosurgery & $1(7.1)$ \\
\hline Follow-up period (months) & $94.2(5-251)$ \\
\hline
\end{tabular}

Values are presented as mean (range) or number (\%). TSH : thyroidstimulating hormone, DI : diabetes insipidus 
TSH-Secreting Pituitary Adenoma | Byun J, et al.

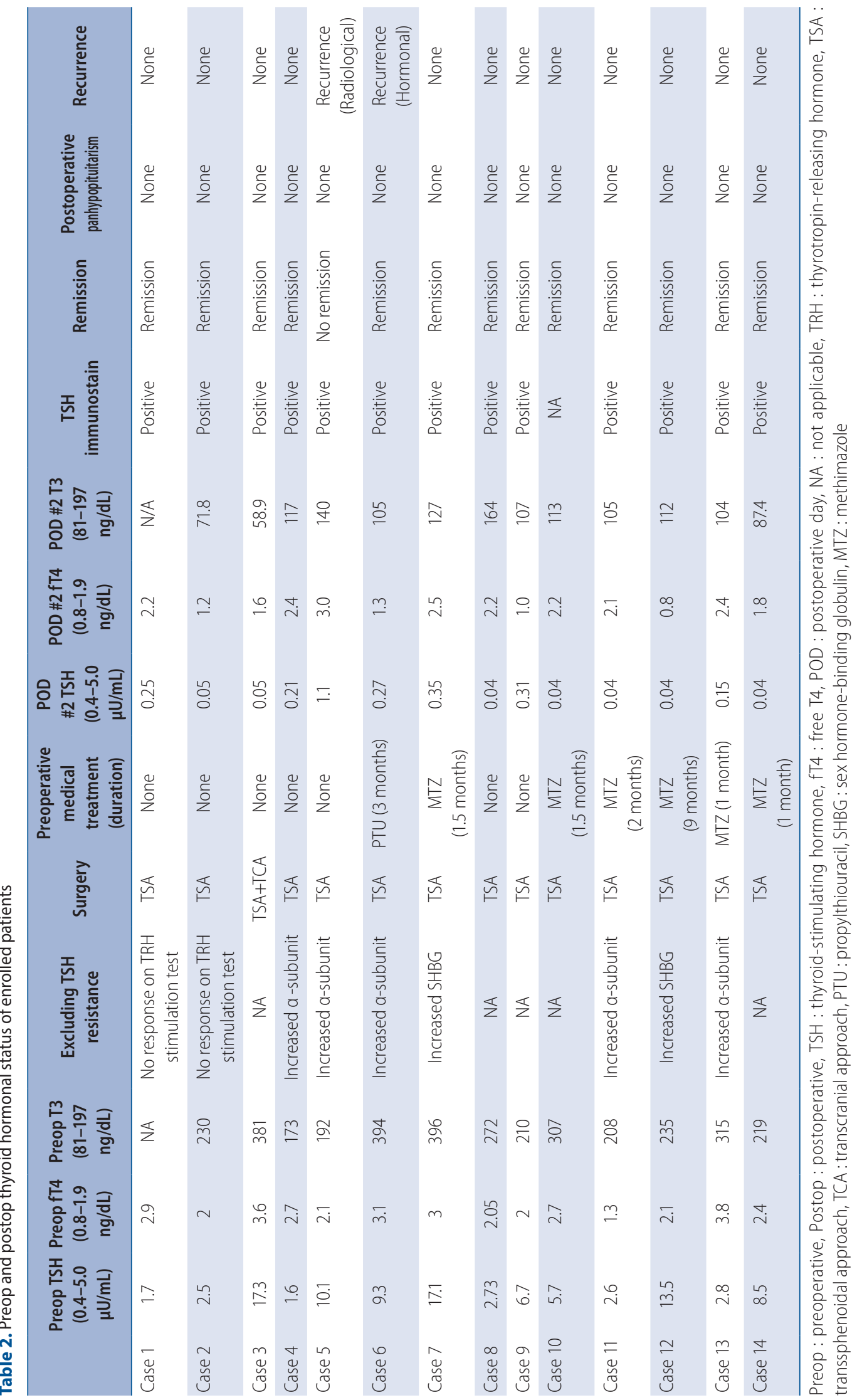



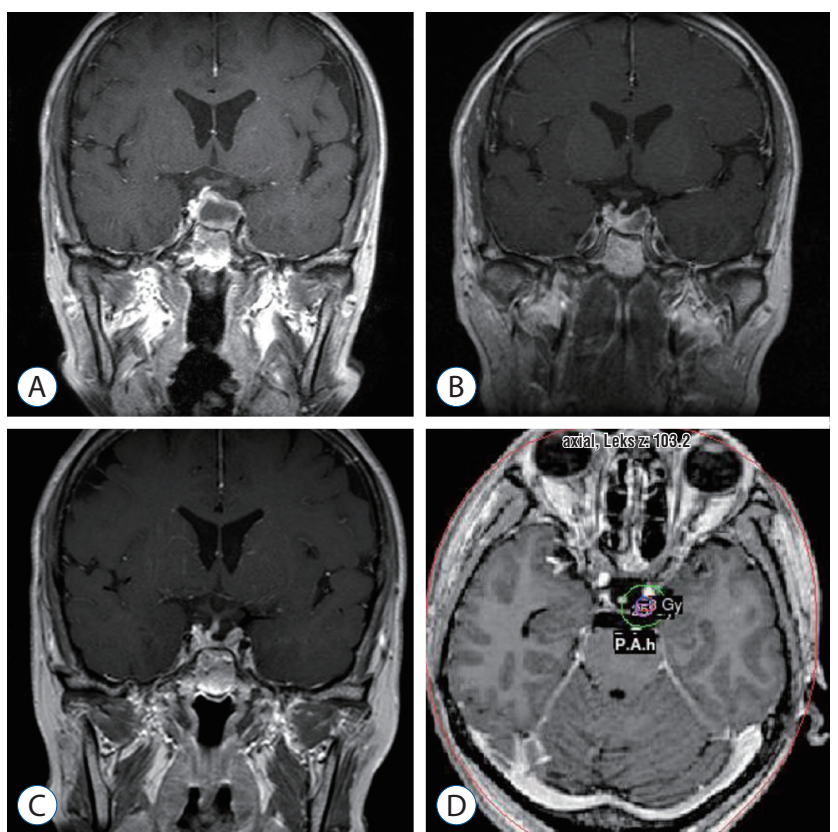

Fig. 4. A case of recurrent TSH-secreting pituitary adenoma. A : A 46-yearold female patient with a tumor located on left side of the sella and deviated pituitary stalk on brain MRI. The patient underwent transnasal transsphenoidal surgery and experienced hormonal remission after surgery. B : Follow-up MRI performed at postoperative 3 months showed no evidence of residual tumor. $C$ and $D$ : Gamma knife radiosurgery planning image; irradiation field included sella and left cavernous sinus. $\mathrm{TSH}$ : thyroid-stimulating hormone, MRI : magnetic resonance imaging.

TSH and alpha subunit with no definite lesion on the thyroid ultrasonograph and brain MRI. The irradiated field included sella and left cavernous sinus targeting microscopic infiltrative lesions which are invisible on brain MRI (Fig. 4). However, this patient has not shown hormonal remission in 6 years after GKRS and is still receiving medical therapy. Recurrence-free survival data are presented in Fig. 5.

The enrolled patients' preoperative and postoperative hormonal statuses are summarized in Table 2. Results of the laboratory work-up which excluded TSH resistance are also presented in Table 2. The treatments and outcomes of recurrent TSH-PA patients are summarized in Table 3.

\section{DISCUSSION}

Owing to its rarity, early recognition of TSH-PA is difficult. We have followed the diagnostic criteria of European Thyroid Association ${ }^{2,13,18}$. If the diagnosis is unclear, a TRH-stimulation test and detection of $\alpha$-subunit are helpful ${ }^{19)}$. However, in

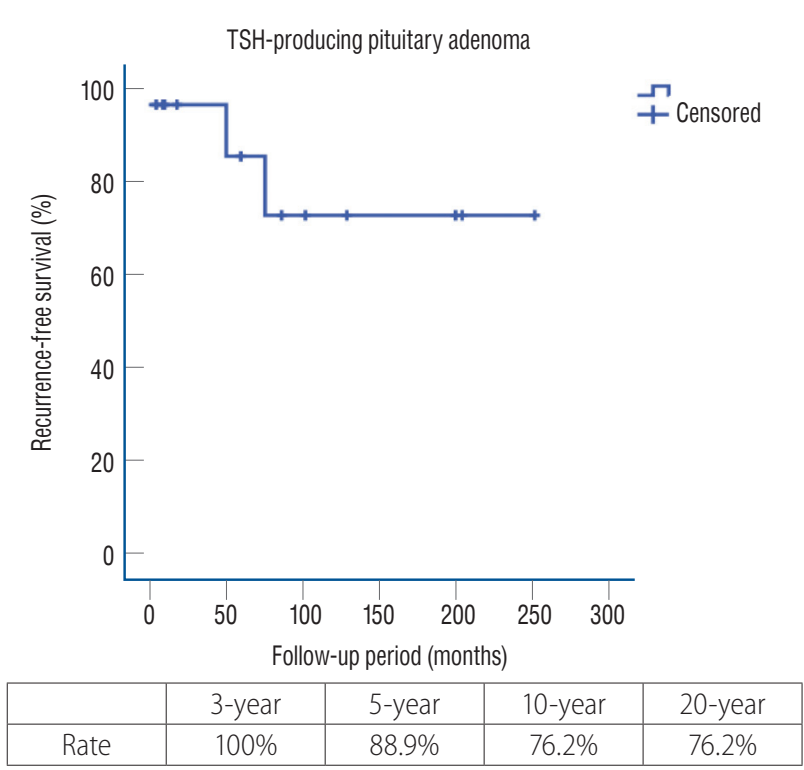

Fig. 5. Kaplan-Meier analysis of recurrence-free survival. Recurrence-free survival of enrolled patients. The 5-year and 20-year recurrence-free survival rates were $88.9 \%$ and $76.2 \%$, respectively. TSH : thyroid-stimulating hormone.

the era of high accessibility with brain MRI, these additional laboratory studies are no longer routinely needed.

TSH-PA is known to present large, invasive tumors that have a relatively hard and fibrotic consistency compared with other functioning pituitary tumors ${ }^{3,4}$. However, in our study, although there were only three microadenomas (maximal diameter $<1 \mathrm{~cm}$ ), the mean tumor size was small, and there was no cavernous sinus invasion of the tumor. Given the widespread use and low cost of brain MRI under the wide coverage of the national health insurance system of South Korea, clinicians can recognize TSH-PA earlier. This may have helped in the identification of small and non-invasive tumors in our series.

The treatment options of functioning PA include surgery, radiosurgery, radiation therapy, and medical therapy. However, the efficacy of each treatment of TSH-PA has not been fully revealed because of its extremely low incidence. Based on the previously reported remission rate of surgery alone for TSH$\mathrm{PA}$, surgical removal is not fully advocated as a first-line treatment. The efficacy of medical treatment for TSH-PA was reported. Beck-Peccoz and Persani ${ }^{2}$ reported a good outcome of medical treatment using somatostatin analog; they reported a rate of a euthyroid state after medical therapy only for TSHPA of $90 \%$ and that one-third of patients experienced a de- 
Table 3. Treatment and outcomes of recurrent TSH-secreting pituitary adenomas

\begin{tabular}{|c|c|c|c|c|c|c|}
\hline & Time to recurrence & MRI findings & $\begin{array}{l}\text { Treatment of } \\
\text { recurrence }\end{array}$ & $\begin{array}{c}\text { Remission after } \\
\text { recurrence } \\
\text { treatment }\end{array}$ & Follow-up period & Note \\
\hline Case 5 & $\begin{array}{l}75 \text { months (radiological } \\
\text { recurrence) }\end{array}$ & Recurrent tumor & Revision TSA & Remission & 156 months & $\begin{array}{l}\text { Patient did not attend } \\
\text { clinic regularly after } \\
\text { 1st surgery }\end{array}$ \\
\hline Case 6 & $\begin{array}{l}50 \text { months } \\
\text { (hormonal recurrence) }\end{array}$ & No lesion & GK radiosurgery & No remission & 149 months & $\begin{array}{l}\text { Ongoing methimazole } \\
\text { therapy }\end{array}$ \\
\hline
\end{tabular}

TSH : thyroid-stimulating hormone, MRI : magnetic resonance imaging, TSA : transsphenoidal approach, GK : gamma knife

crease in tumor size. However, medical therapy requires frequent clinical visits, lifelong treatment, and high cost. Moreover, its long-term durability has not been elucidated.

Recently, a relatively good outcome of the surgical removal of TSH-PA has been reported. Yamada et al. ${ }^{20)}$ reported a remission rate of $84 \%$ for surgery for TSH-PA, whereas Rotermund et al. ${ }^{16)}$ also reported a remission rate of surgery alone for TSH-PA of $91.7 \%$ in their case series. In our series, the remission rate of TSH-PA after surgical treatment alone was 92.3\%. TSH-PA tumor is known to be relatively hard and fibrotic, which can make surgical removal difficult ${ }^{4,20)}$. Our surgeons agreed over the finding of a relatively hard consistency of TSH-PA tumors, but this may not be a major obstacle to complete tumor removal. In addition, the cleavage plane of the tumor was sometimes identified, which facilitated en-bloc tumor removal. To the best of our knowledge, our endocrinological outcome of surgery alone for TSH-PA is the best treatment outcome reported to date. The relatively small size (mean, $17.1 \mathrm{~mm}$ ) of tumors without revised Knosp grade IV were included this study; it may have contributed to the excellent remission rate of surgery alone for TSH-PA.

Perioperative hyperthyroidism can provoke atrial fibrillation and increased cardiac output through positive inotropic and chronotropic effects with water retention ${ }^{15}$. We used an antithyroid drug to decrease the thyroid hormone levels in the preoperative period. We preferred methimazole to propylthiouracil because it can achieve a euthyroid state more rapidly ${ }^{15}$. An arterial catheter was also inserted during surgery in every patient for the meticulous monitoring of changes in blood pressure. Thus, there was no patient who experienced perioperative thyroid storm or cardiovascular complications in our series.

Stereotactic radiosurgery (SRS) is considered as one treatment option for functioning and non-functioning PA. In a re- cent review article by Minnitia et al. ${ }^{10)}$, the long-term tumor control rate of SRS ranged from $85 \%$ to $100 \%$ and the endocrinological remission rate was about $45-50 \%$. Although we did not consider SRS as initial treatment, SRS could be used as salvage therapy for recurrent TSH-PA. In our study, one patient with recurrence received GKRS; this patient showed an elevated TSH level at the 4-year follow-up after the first surgery. However, there was no definite visible recurrent lesion on brain MRI, so the patient received GKRS in the sella fossa and left cavernous sinus targeting microscopic infiltration. Unfortunately, endocrinological remission was not achieved 4 years after GKRS. The effect of SRS has been reported in the treatment of recurrent functioning $\mathrm{PA}^{5,9)}$. Mouslech et al. ${ }^{12)}$ reported a case of endocrinological remission of GKRS for recurrent TSH-PA. They reviewed 14 cases of TSH-PA treated by GKRS; over $80 \%$ of cases showed endocrinological remission after GKRS ${ }^{12)}$. Although one patient of our series did not show a good outcome after GKRS, according to a previous report, GKRS may be an effective option for recurrent or residual TSH-PA.

There has been a debate on the treatment of recurrent TSHPA. We considered redo-surgery as a first-line treatment if recurrent tumor is resectable. Both somatostatin analog medical therapy and SRS could be good treatment options for recurrent TSH-PA. There is no evidence of superiority between medical therapy and SRS. Medical therapy showed a favorable outcome; however, it has side effect such as gastrointestinal disturbance and glucose intolerance and requires frequent clinical visits ${ }^{11}$. Although radiosurgery is associated with a risk of radiation injury, SRS can be a curative treatment. In our institution, we preferred SRS to medical therapy in treatment of recurrent TSH-PA.

Yamada et al. ${ }^{20)}$ reported that cavernous sinus invasion and maximal tumor diameter are predictors of good surgical out- 
comes of TSH-PA. We failed to analyze the prognostic factors of postoperative endocrinological outcome because there was only one case without remission. However, in our series, the postoperative day $2 \mathrm{TSH}$ level in all patients in remission was lower than the normal range (less than $0.4 \mu \mathrm{U} / \mathrm{mL}$ ), whereas the patient without remission showed a TSH level in the normal range. The half-life of TSH is about 1 hour, whereas that of fT4 is approximately 1 week. Thus, an immediate decrease of the functioning tumor burden of TSH-PA may be reflected in TSH rather than in fT4. A subnormal level of TSH in the immediate postoperative period may be a predictor of endocrinological remission of TSH-PA. A future study should be conducted to identify the predictors of endocrinological remission and efficacy of SRS for TSH-PA.

\section{Limitations and strengths}

Our retrospective study included only 14 patients with TSH-PA, which precluded a meaningful multivariate analysis of the prognostic factors for endocrinological remission along with the limitations inherent to a retrospective study. In addition, this study spanned over 20 years and we have not performed consistent preoperative laboratory work-up. We are not clearly aware of the effect of the preoperative antithyroid hormone on the postoperative evaluation of TSH, fT4, and T3; pituitary function is usually unstable for several months after surgery. TSH-PA is an extremely rare tumor and its early diagnosis is difficult in practice.

To the best of our knowledge, we have here reported the best surgical outcome for such cases (remission rate of $92.8 \%$ ). Although medical therapy using somatostatin analogs and radiosurgery is a possible treatment option and has the advantage of non-invasiveness, surgery is a simple effective treatment and could provide the best outcome for TSH-PA.

\section{CONCLUSION}

TSH-PA is an extremely rare functioning pituitary tumor. Early suspicion and establishment of the diagnosis of TSH-PA may be extremely important. Surgical treatment of TSH-PA showed excellent surgical outcomes. The TSH level in the immediate postoperative period may be a predictor of endocrinological remission. A future multi-center investigation of such cases is needed.

\section{CONFLICTS OF INTEREST}

No potential conflict of interest relevant to this article was reported.

\section{INFORMED CONSENT}

This type of study does not require informed consent.

\section{AUTHOR CONTRIBUTIONS}

\author{
Conceptualization : JB, JHK \\ Data curation : JB \\ Formal analysis : JB \\ Methodology : JB, YHK \\ Project administration : JB, YHC \\ Visualization: JB, SHH \\ Writing - original draft : JB, JHK \\ Writing - review \& editing: YHK, YHC, CJK
}

\section{ORCID}

$\begin{array}{ll}\text { Joonho Byun } & \text { https://orcid.org/0000-0003-0687-3286 } \\ \text { Jeong Hoon Kim } & \text { https://orcid.org/0000-0001-7385-2368 } \\ \text { Young-Hoon Kim } & \text { https://orcid.org/0000-0002-8852-6503 } \\ \text { Young Hyun Cho } & \text { https://orcid.org/0000-0002-3274-5096 } \\ \text { Seok Ho Hong } & \text { https://orcid.org/0000-0003-4349-7021 } \\ \text { Chang Jin Kim } & \text { https://orcid.org/0000-0003-2036-8294 }\end{array}$

\section{- Acknowledgements}

We are indebted to endocrinologists at our institute who diagnosed and referred patients to us. We appreciate their efforts for the consultation and collaborative management of neurosurgical patients. We also thank Min Ji Kim, a neurologist in Daejeon, Jin Kyu Kim, a neurosurgeon in Mokpo, who gave us warm advice for manuscript. 


\section{References}

1. Beck-Peccoz P, Lania A, Beckers A, Chatterjee K, Wemeau JL : 2013 European thyroid association guidelines for the diagnosis and treatment of thyrotropin-secreting pituitary tumors. Eur Thyroid J 2 : 76-82, 2013

2. Beck-Peccoz $P$, Persani $L$ : Medical management of thyrotropin-secreting pituitary adenomas. Pituitary $5: 83-88,2002$

3. Brucker-Davis F, Oldfield EH, Skarulis MC, Doppman JL, Weintraub BD : Thyrotropin-secreting pituitary tumors: diagnostic criteria, thyroid hormone sensitivity, and treatment outcome in 25 patients followed at the National Institutes of Health. J Clin Endocrinol Metab 84 : 476-486, 1999

4. Ezzat S, Horvath E, Kovacs K, Smyth HS, Singer W, Asa SL : Basic fibroblast growth factor expression by two prolactin and thyrotropinproducing pituitary adenomas. Endocr Pathol 6 : 125-134, 1995

5. Hughes JD, Young WF, Chang AY, Link MJ, Garces YI, Laack NN, et al. : Radiosurgical management of patients with persistent or recurrent cushing disease after prior transsphenoidal surgery: a management algorithm based on a 25-year experience. Neurosurgery 86 : 557-564, 2020

6. Knosp E, Steiner E, Kitz K, Matula C : Pituitary adenomas with invasion of the cavernous sinus space: a magnetic resonance imaging classification compared with surgical findings. Neurosurgery 33 : 610-617; discussion 617-618, 1993

7. Macchia E, Gasperi M, Lombardi M, Morselli L, Pinchera A, Acerbi G, et al. : Clinical aspects and therapeutic outcome in thyrotropin-secreting pituitary adenomas: a single center experience. J Endocrinol Invest 32 : 773-779, 2009

8. Micko AS, Wöhrer A, Wolfsberger S, Knosp E : Invasion of the cavernous sinus space in pituitary adenomas: endoscopic verification and its correlation with an MRI-based classification. J Neurosurg 122 : 803-811, 2015

9. Minniti G, Osti MF, Niyazi M : Target delineation and optimal radiosurgical dose for pituitary tumors. Radiat Oncol 11 : 135, 2016

10. Minnitia G, Clarkea E, Scaringia C, Enrici RM : Stereotactic radiotherapy and radiosurgery for non-functioning and secreting pituitary adenomas. Rep Pract Oncol Radiother 21 : 370-378, 2016
11. Mouslech Z, Somali M, Sakali AK, Savopoulos C, Mastorakos G, Hatzitolios Al : TSH-secreting pituitary adenomas treated by gamma knife radiosurgery: our case experience and a review of the literature. Hormones (Athens) $15:$ 122-128, 2016

12. Mouslech Z, Somali M, Sakali AK, Savopoulos C, Mastorakos G, Hatzitolios Al : TSH-secreting pituitary adenomas treated by gamma knife radiosurgery: our case experience and a review of the literature. Hormones 15 : 122-128, 2016

13. Ónnestam L, Berinder $K$, Burman $P$, Dahlqvist $P$, EdenEngström B, Wahlberg J, et al. : National incidence and prevalence of TSH-secreting pituitary adenomas in Sweden. J Clin Endocrinol Metab 98 : 626-635, 2013

14. Ostrom QT, Gittleman H, Fulop J, Liu M, Blanda R, Kromer C, et al. : CBTRUS statistical report: primary brain and central nervous system tumors diagnosed in the United States in 2008-2012. Neuro Oncol 17 Suppl 4 : iv1-iv62, 2015

15. Palace MR : Perioperative management of thyroid dysfunction. Health Serv Insights 10 : 1178632916689677,2017

16. Rotermund R, Riedel N, Burkhardt T, Matschke J, Schmidt N-O, Aberle J, et al. : Surgical treatment and outcome of TSH-producing pituitary adenomas. Acta Neurochir (Wien) 159 : 1219-1226, 2017

17. Sanno N, Teramoto A, Osamura RY : Long-term surgical outcome in 16 patients with thyrotropin pituitary adenoma. J Neurosurg 93 : 194200, 2000

18. Tjornstrand A, Gunnarsson K, Evert M, Holmberg E, Ragnarsson O, Rosén $T$, et al. : The incidence rate of pituitary adenomas in western Sweden for the period 2001-2011. Eur J Endocrinol 171 : 519-526, 2014

19. Tjörnstrand A, Nyström HF : Diagnostic approach to TSH-producing pituitary adenoma. Eur J Endocrinol 177 : R183-R197, 2017

20. Yamada S, Fukuhara N, Horiguchi K, Yamaguchi-Okada M, Nishioka H, Takeshita A, et al. : Clinicopathological characteristics and therapeutic outcomes in thyrotropin-secreting pituitary adenomas: a single-center study of 90 cases. J Neurosurg 121 : 1462-1473, 2014

21. Zhao W, Ye H, Li Y, Zhou L, Lu B, Zhang S, et al. : Thyrotropin-secreting pituitary adenomas: diagnosis and management of patients from one Chinese center. Wien Klin Wochenschr 124 : 678-684, 2012 